\title{
Expression of cancer-testis antigens in endometrial carcinomas using a tissue microarray
}

\author{
Dhananjay A Chitale ${ }^{1}$, Achim A Jungbluth ${ }^{2}$, David S Marshall ${ }^{1}$, Mario M Leitao ${ }^{3}$, \\ Cyrus V Hedvat ${ }^{1}$, Denise Kolb ${ }^{1}$, Giulio C Spagnoli ${ }^{4}$, Kristin Iversen ${ }^{1}$ and Robert A Soslow ${ }^{1}$ \\ ${ }^{1}$ Department of Pathology, Memorial Sloan-Kettering Cancer Center, New York, NY, USA; ${ }^{2}$ Ludwig Institute \\ for Cancer Research, New York, NY, USA; ${ }^{3}$ Gynecology Service, Department of Surgery, Memorial \\ Sloan-Kettering Cancer Center, New York, NY, USA and ${ }^{4}$ Department of Surgery, University Hospital, \\ Basel, Switzerland
}

\begin{abstract}
Cancer-testis (CT) antigens are expressed in a variety of malignant tumors, but in normal adult tissue, they are only expressed in testicular germ cells. Owing to this tumor-associated expression pattern, these antigens are of major interest as potential targets for immunotherapy and possibly for diagnostic purposes. This study was performed to analyze the expression of four CT antigens, NY-ESO-1, MAGE-A3, MAGE-A4, and CT7/MAGE-C1, in endometrial carcinoma using immunohistochemistry, and to correlate expression with histologic subtypes, grade, and expression of WT1 and p53. Formalin-fixed paraffin-embedded tissues of 130 endometrial carcinomas of the following types and grades were analyzed using a tissue microarray: 85 endometrioid carcinomas (FIGO grade 1, 39; grade 2, 11; and grade 3, 35), 18 papillary serous carcinomas, 12 clear cell carcinomas, 13 malignant mixed mullerian tumors, one mucinous adenocarcinoma, and one undifferentiated carcinoma. The following anti-CT monoclonal antibodies/antigens were studied by immunohistochemistry: monoclonal antibody ES121/NY-ESO-1, monoclonal antibody M3H67/MAGE-A3, monoclonal antibody 57B/ MAGE-A4, and monoclonal antibody CT7-33/CT7. The CT expression data were compared to WT1 and p53 protein expression as analyzed in a previous study. Positive staining with anti-CT monoclonal antibodies was graded as follows: focal, $<5 \%$ positive cells; $1+, 5-25 \%$ cells; $2+, 26-50 \%$ cells; $3+, 51-75 \%$; and $4+,>75 \%$ cells. The $3+$ and $4+$ staining patterns were considered homogeneous patterns of potential clinical significance and were scored positive for statistical analysis. In low-grade tumors, the most immunoreactivity was seen with mAb M3H67 but little labeling was observed with the other monoclonal antibodies. In high-grade tumors, monoclonal antibodies M3H67 (25\%), 57B (23\%), and CT7-33 (20\%) showed the highest reactivity, while ES121 showed the lowest immunoreactivity (6\%). The staining pattern was mostly heterogeneous. Statistical significance was found solely for the correlation of monoclonal antibody $57 \mathrm{~B}$ staining and p53 expression. No correlation was found for any anti-CT monoclonal antibody staining and clinical stage or for anti-CT staining and WT1 expression. CT antigens CT7, MAGE-A3 and MAGE-A4, but not NY-ESO-1, are expressed in high-grade endometrial carcinomas, and expression of MAGE-A4 is correlated with the presence of overexpressed p53.
\end{abstract}

Modern Pathology (2005) 18, 119-126, advance online publication, 23 July 2004; doi:10.1038/modpathol.3800232

Keywords: CT antigen; endometrial cancer; immunotherapy; NY-ESO-1

Cancer-testis (CT) antigens are expressed in a variety of malignant tumors, but in normal adult tissue, they are only expressed in testicular germ cells. They are occasionally found in placental

Correspondence: Dr RA Soslow, MD, Department of Pathology, Memorial Sloan-Kettering Cancer Center, 1275 York Avenue, New York, NY 10021, USA.

E-mail: soslowr@mskcc.org

Received 14 April 2004; revised and accepted 1 June 2004; published online 23 July 2004 tissue as well. ${ }^{1} \mathrm{CT}$ antigens have therefore become a major focus as potential targets for immunotherapy and possibly for diagnosis. CT antigens have been isolated by techniques using their ability to elicit autologous cellular and humoral immune responses in cancer patients. ${ }^{2-4}$

To date, more than 30 genes and gene families, such as MAGE, BAGE, GAGE, NY-ESO-1, and CT7 have been isolated. ${ }^{5}$ While the first CT antigens, MAGE, BAGE, and GAGE, were identified by autologous T-cell epitope cloning, ${ }^{6-9}$ others were 
isolated by the SEREX (serological analysis of recombinant tumor cDNA expression libraries with autologous serum) method ${ }^{10,11}$ or by representational difference analysis. ${ }^{12,13}$ MAGE expression has been found in a wide array of neoplastic lesions and is most prevalent in malignant melanomas, several types of carcinomas, and certain sarcomas. ${ }^{14-20}$ NY-ESO- $1^{21,22}$ has been isolated in esophageal carcinoma but has also been found in several other malignancies, such as melanoma; neuroblastoma; carcinoma of the ovary, breast, and lung; and in synovial sarcoma. ${ }^{23-29}$

The CT7 antigen was identified by the SEREX approach using a melanoma cell line SK-MEL37 and allogeneic sera from a melanoma patient. ${ }^{30} C T 7$ is identical to $M A G E-C 1$, identified independently by another group using representational difference analysis. ${ }^{13}$

Since earlier studies were mainly based on RTPCR analyses, our group and others generated monoclonal antibodies (mAbs) to several CT antigens in order to investigate their expression pattern on a protein level. Although several tumor types have been analyzed for CT antigen expression, little is known about CT antigen expression in endometrial carcinomas; only one previous study has addressed this issue. ${ }^{31}$ Several studies have demonstrated the importance of p53 as a prognostic marker in endometrial carcinoma. ${ }^{32-34}$ The Wilms' tumor gene (WT1) shows a peculiar expression pattern in normal endometrium..$^{35}$ The WT1 protein expression in endometrial carcinomas and its potential correlation with the expression of other proteins has not been analyzed. We previously analyzed the expression of WT1 and $\mathrm{p} 3^{36}$ in a panel of endometrial carcinomas on a protein level. In the current study, this panel of endometrial carcinomas was used to analyze the expression of four CT antigens by immunohistochemistry employing tissue microarray technology and previously generated mAb ES121 to NY-ESO-1, mAb 57B to MAGE-A4, mAb M3H67 to MAGE-A3, and mAb CT7-33 to CT7. ${ }^{15,16,23,37}$ We also correlated CT antigen expression with clinical stage, histologic subtype, tumor grade, and expression of WT1 and p53, as previously studied. $^{36}$

\section{Materials and methods}

\section{Case Selection}

Formalin-fixed paraffin-embedded tissues of 130 endometrial carcinoma cases were obtained from the archives of the Department of Pathology, Memorial Sloan-Kettering Cancer Center. These consisted of 85 endometrioid adenocarcinomas, 18 papillary serous carcinomas, 12 clear cell carcinomas, 13 malignant mixed mullerian tumors, one mucinous adenocarcinoma, and one undifferentiated carcinoma. The 85 endometrioid adenocarcinomas were graded according to the International Federation of Gynecology and Obstetrics (FIGO) classification system as follows: grade 1, 39; grade 2, 11 ; and grade 3,35 . For the purposes of simplifying presentation of the data, we considered grade 1 and 2 endometrioid adenocarcinomas (including the mucinous adenocarcinoma) as low-grade and grade 3 endometrioid adenocarcinomas, papillary serous carcinomas, clear cell carcinomas, and malignant mixed mullerian tumors as high grade. Five cases of clear cell carcinoma and seven papillary serous carcinomas showed areas of endometrioid or mucinous differentiation, each forming less than $10 \%$ of the tumor. One case of malignant mixed mullerian tumor displayed grade 3 endometrioid adenocarcinoma areas, and one had a focal rhabomyosarcomatous component. The other malignant mixed mullerian tumors contained high-grade epithelial (primarily serous) and sarcomatous components. Slides from all cases were reviewed and representative blocks chosen for tissue microarray assembly.

\section{Immunohistochemistry}

The mAbs used in this study have been described before and are listed in Table 1. ${ }^{16,21,23,30,37-39}$ Commercially available antibodies to WT1 (Santa Cruz; Santa Cruz, CA, USA) and p53 (Clone D07 (DAKO; Carpinteria, CA, USA)) were used. A heat-based antigen-retrieval method was used for all antibodies (vegetable steamer (Black and Decker; Denver, CO, USA); $\left.90^{\circ} \mathrm{C}, 30 \mathrm{~min}\right)$. A biotinylated horse antimouse secondary antibody (1:200; Vector (Burlingame, CA, USA)) was used to detect primary

Table $1 \mathrm{mAbs}$ for immunohistochemical analysis

\begin{tabular}{lllll}
\hline Monoclonal antibody & Antigen & Source & Cellular staining pattern & References \\
\hline ES121 & NY-ESO-1 & LICR & Nuclear \& cytoplasmic & 21,23 \\
57B & MAGE-A4 & Dr Spagnoli, Basel, Switzerland & Nuclear \& cytoplasmic & $16,38,39$ \\
M3H67 & MAGE-A3 & LICR & Nuclear \& cytoplasmic & Manuscript in preparation \\
CT7-33 & CT7 (MAGE-C1) & LICR & Nuclear \& cytoplasmic & 30,37 \\
WT1 & WT1 & Santa Cruz; & Nuclear & \\
p53 & p53 & Santa Cruz, CA & Dako; Carpinteria, CA; Clone D07 & Nuclear \\
\hline
\end{tabular}

LICR, Ludwig Institute of Cancer Research. 
antibody, followed by an avidin-biotin system (ABC-elite kit, Vector). 3,3'-Diaminobenzidine tetrahydrochloride (Biogenex; San Ramon, CA, USA) served as chromogen. Endogenous peroxidase was suppressed by $1 \% \mathrm{H}_{2} \mathrm{O}_{2}$ for $20 \mathrm{~min}$. The extent of tumor staining was estimated on the basis of numbers of tumor cells stained and graded as follows: Focal, approximately $<5 \%$; , $5-25 \%$; ++ , $26-50 \%$;,$+++ 51-75 \%$; and,$++++>75 \%$. Staining in $<50 \%$ of the tumor $(+$ to ++ ) was considered heterogeneous staining. Testis with preserved spermatogenesis was used as a positive control for all antibodies.

\section{Tumor Microarray}

Core-needle biopsies of paraffin-embedded tissue were obtained and then re-embedded in an array master block using techniques originally developed by Kononen et $a l^{40}$ and then modified by Hedvat et al. $^{41}$ A Beecher Instruments (Sun Prairie, WI, USA) arraying device to produce sample circular spots $(0.6 \mathrm{~mm}$ diameter) was used to assemble the arrays. At least two representative areas and companion tissue specimens were included from each case on the tissue microarray block. Also included were tissue samples from normal liver, kidney, and proliferative endometrium, as normal controls. The tissue microarray block was then used to perform immunohistochemical analysis. Three pathologists (DAC, RAS, AAJ) evaluated the immunohistochemistry slides independently, without knowledge of clinical or histologic data.

\section{Statistical Analysis}

Correlation between antigen expression was performed using the $\chi^{2}$-test of significance. Correlation between clinical stage and antigen expression and between pathologic types and antigen expression were also performed using the same statistical test. Two-tailed $P$-values of 0.05 or less were considered statistically significant. All statistical analyses were performed using the SAS Software (SAS Institute Inc.; Cary, NC, USA).

\section{Results}

The result of the immunohistochemical analysis of the 130 cases of endometrial cancer is listed in Tables 2-7. No staining was observed in any normal tissue present in our arrays (kidney, liver, and nonneoplastic uterus). Taken together, there was little immunoreactivity with our anti-CT mAbs in lowgrade endometrial carcinomas. This group consisted of 50 cases of endometrioid adenocarcinomas (grade 1, 39; grade 2, 11) and one case of mucinous adenocarcinoma. Among these low-grade cases, highest expression was seen with mAb M3H67 (Figure 1). Surprisingly, in low-grade tumors, staining was seen almost exclusively in grade 1 cases, while grade 2 tumors were negative in all except 1 of 11 CT7-33-positive cases. In high-grade endometrial carcinomas, more substantial immunoreactivity was observed for all anti-CT mAbs, except for ES121 (Figure 2). This held true regardless of whether grade 3 endometrioid adenocarcinomas were analyzed separately or in combination with the other high-grade endometrial carcinomas. The highest expression among high-grade endometrial carcinomas was seen with mAb M3H67 (25\%), while mAb ES121 showed the lowest immunoreactivity (6\%). The incidence of immunostaining with mAb 57B $(23 \%)$ and mAb CT7-33 $(20 \%)$ was similar. The immunoreactivity pattern was almost exclusively heterogeneous for all mAbs. However, homogeneous staining (ie, staining in more than $50 \%$ of the tumor) was seen with ES121 in a single case of grade 3 endometrioid adenocarcinoma FIGO 3 and in several cases of papillary serous carcinoma and

Table 2 Summary of distribution of cases with positive expression with different antibodies

\begin{tabular}{|c|c|c|c|c|c|c|c|}
\hline & Diagnosis & $M A G E-4(57 B)$ & $M A G E-3(M 3 H 67)$ & CT7 (CT7-33) & NY-ESO-1 (ES121) & $W T 1$ & p53 \\
\hline \multirow[t]{3}{*}{ Low-grade EMCs } & EMOC FIGO 1 & $1 / 39(2.5 \%)$ & 6/39 (15\%) & $1 / 39(2.5 \%)$ & $2 / 39(5 \%)$ & $5 / 39(13 \%)$ & $1 / 39(2.5 \%)$ \\
\hline & EMOC FIGO 2 & $0 / 11(0 \%)$ & $0 / 11(0 \%)$ & $1 / 11(9 \%)$ & $0 / 11(0 \%)$ & $1 / 11(9 \%)$ & $0 / 11(0 \%)$ \\
\hline & MCA & $0 / 1$ & $0 / 1$ & $0 / 1$ & $0 / 1$ & $0 / 1$ & $0 / 1$ \\
\hline Total low grade & & $1 / 51(2 \%)$ & 6/51 (12\%) & $2 / 51(4 \%)$ & $2 / 51(4 \%)$ & $6 / 51(12 \%)$ & $1 / 51(2 \%)$ \\
\hline \multirow[t]{5}{*}{ High-grade EMCs } & PSC & $9 / 18(50 \%)$ & 7/18 (39\%) & $4 / 18(22 \%)$ & $1 / 18(5.5 \%)$ & $7 / 18(39 \%)$ & $9 / 18(50 \%)$ \\
\hline & MMMT & $2 / 13(15 \%)$ & $3 / 13(24 \%)$ & $4 / 13(31 \%)$ & $1 / 13(8 \%)$ & $7 / 13(54 \%)$ & $8 / 13(62 \%)$ \\
\hline & EMOC FIGO 3 & $6 / 35(17 \%)$ & $9 / 35(26 \%)$ & $8 / 35(23 \%)$ & $3 / 35(9 \%)$ & $11 / 35(31 \%)$ & $7 / 35(20 \%)$ \\
\hline & CC & $1 / 12(8 \%)$ & $1 / 12(8 \%)$ & $0 / 12(0 \%)$ & $0 / 12(0 \%)$ & $2 / 12(17 \%)$ & $2 / 12(17 \%)$ \\
\hline & UCA & $0 / 1$ & $0 / 1$ & $0 / 1$ & $0 / 1$ & $0 / 1$ & $0 / 1$ \\
\hline Total high grade & & $18 / 79(23 \%)$ & $20 / 79(25 \%)$ & 16/79 (20\%) & $5 / 79(6 \%)$ & $27 / 79(34 \%)$ & $26 / 79(33 \%)$ \\
\hline Total positive & & $19 / 130(15 \%)$ & $26 / 130(20 \%)$ & $18 / 130(14 \%)$ & $7 / 130(5 \%)$ & $34 / 130(26 \%)$ & $27 / 130(21 \%)$ \\
\hline
\end{tabular}

EMC, endometrial carcinoma; EMOC, endometrioid carcinoma; MCA, mucinous adenocarcinoma; PSC, papillary serous carcinoma; MMMT, malignant mixed mullerian tumor; CC, clear cell; UCA, undifferentiated carcinoma. 
Table 3 Distribution of cases with positive expression of NY-ESO-1 (mAb ES121)

\begin{tabular}{lccccc}
\hline Diagnosis & $\begin{array}{c}\text { Total } \\
\text { positive }\end{array}$ & + & ++ & +++ & ++++ \\
\hline Papillary serous carcinoma & $1 / 18$ & 0 & 1 & 0 & 0 \\
Malignant mixed mullerian tumor & $1 / 13$ & 0 & 1 & 0 & 0 \\
Clear cell carcinoma & $0 / 12$ & 0 & 0 & 0 & 0 \\
Undifferentiated carcinoma & $0 / 1$ & 0 & 0 & 0 & 0 \\
EMOC FIGO 3 & $3 / 35$ & 1 & 0 & 2 & 0 \\
EMOC FIGO 2 & $0 / 11$ & 0 & 0 & 0 & 0 \\
EMOC FIGO 1 & $2 / 39$ & 2 & 0 & 0 & 0 \\
Mucinous carcinoma & $0 / 1$ & 0 & 0 & 0 & 0 \\
\hline
\end{tabular}

EMOC, endometrioid carcinoma.

Table 4 Distribution of cases with positive expression of MAGE-A4 (mAb 57B)

\begin{tabular}{lrrrrr}
\hline Diagnosis & $\begin{array}{c}\text { Total } \\
\text { positive }\end{array}$ & + & ++ & +++ & ++++ \\
\hline Papillary serous carcinoma & $9 / 18$ & 3 & 0 & 1 & 5 \\
Malignant mixed mullerian tumor & $2 / 13$ & 1 & 0 & 0 & 1 \\
Clear cell carcinoma & $1 / 12$ & 1 & 0 & 0 & 0 \\
Undifferentiated carcinoma & $0 / 1$ & 0 & 0 & 0 & 0 \\
EMOC FIGO 3 & $6 / 35$ & 1 & 2 & 1 & 2 \\
EMOC FIGO 2 & $0 / 11$ & 0 & 0 & 0 & 0 \\
EMOC FIGO 1 & $1 / 39$ & 0 & 1 & 0 & 0 \\
Mucinous carcinoma & $0 / 1$ & 0 & 0 & 0 & 0 \\
\hline
\end{tabular}

EMOC, endometrioid carcinoma.

Table 5 Distribution of cases with positive expression of MAGE-A3 (mAb M3H67)

\begin{tabular}{lrrrrr}
\hline Diagnosis & $\begin{array}{c}\text { Total } \\
\text { positive }\end{array}$ & + & ++ & +++ & ++++ \\
\hline Papillary serous carcinoma & $7 / 18$ & 2 & 2 & 0 & 3 \\
Malignant mixed mullerian tumor & $3 / 13$ & 1 & 0 & 0 & 2 \\
Clear cell carcinoma & $1 / 12$ & 1 & 0 & 0 & 0 \\
Undifferentiated carcinoma & $0 / 1$ & 0 & 0 & 0 & 0 \\
EMOC FIGO 3 & $9 / 35$ & 3 & 2 & 2 & 2 \\
EMOC FIGO 2 & $0 / 11$ & 0 & 0 & 0 & 0 \\
EMOC FIGO 1 & $6 / 39$ & 2 & 2 & 2 & 0 \\
Mucinous carcinoma & $0 / 1$ & 0 & 0 & 0 & 0 \\
\hline
\end{tabular}

EMOC, endometrioid carcinoma.
Table 6 Distribution of cases with positive expression of CT7 (mAb CT7-33)

\begin{tabular}{lccccc}
\hline Diagnosis & $\begin{array}{c}\text { Total } \\
\text { positive }\end{array}$ & + & ++ & +++ & ++++ \\
\hline Papillary serous carcinoma & $4 / 18$ & 3 & 1 & 0 & 0 \\
Malignant mixed mullerian tumor & $4 / 13$ & 2 & 1 & 0 & 1 \\
Clear cell carcinoma & $0 / 12$ & 0 & 0 & 0 & 0 \\
Undifferentiated carcinoma & $0 / 1$ & 0 & 0 & 0 & 0 \\
EMOC FIGO 3 & $8 / 35$ & 6 & 2 & 0 & 0 \\
EMOC FIGO 2 & $1 / 11$ & 1 & 0 & 0 & 0 \\
EMOC FIGO 1 & $1 / 39$ & 1 & 0 & 0 & 0 \\
Mucinous carcinoma & $0 / 1$ & 0 & 0 & 0 & 0 \\
& & & & &
\end{tabular}

EMOC, endometrioid carcinoma.

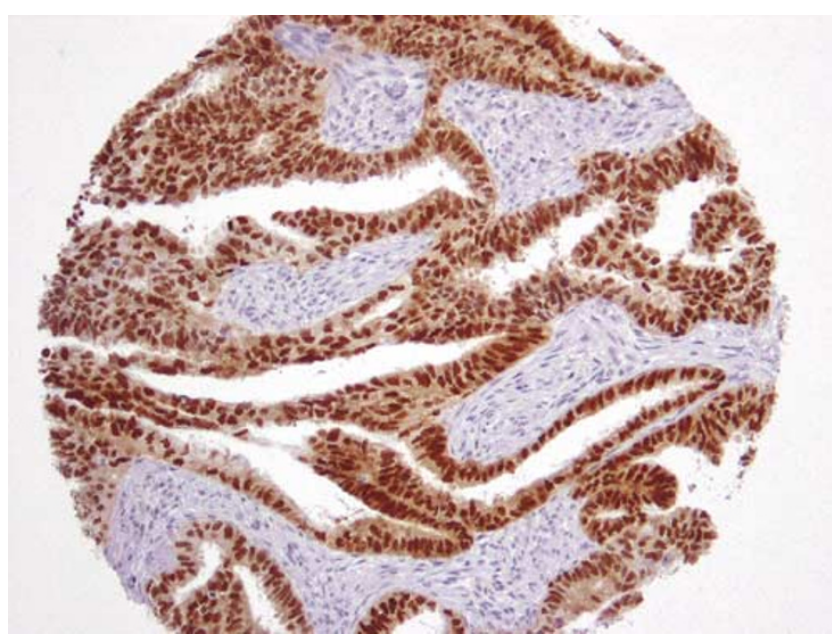

Figure 1 MAGE-A3 in a FIGO grade 1 endometrioid adenocarcinoma.

malignant mixed mullerian tumor for mAbs 57B and M3H67. CT7 staining was almost exclusively heterogeneous, except a single case of ++++ staining. The overall staining of low-grade and high-grade tumors was highest for mAb M3H67 $(20 \%)$ and lowest for mAb ES121 (5\%). No statistically significant difference was found for the correlation of any anti-CT antigen mAb staining and clinical stage.

Table 7 Distribution of cases with positive expression in high-grade vs low-grade endometrial carcinomas

\begin{tabular}{|c|c|c|c|c|c|c|}
\hline & $57 B$ & М3H67 & CT7 & ES121 & p53 & WT1 \\
\hline HG & $18 / 79(23 \%)$ & $\begin{array}{c}20 / 79(25 \%) \\
P=0.0593\end{array}$ & $\begin{array}{c}16 / 79(20 \%) \\
P=0.0129\end{array}$ & $\begin{array}{l}5 / 79(6 \%) \\
P=0.5526\end{array}$ & $\begin{array}{c}27 / 79(34 \%) \\
P<0.001\end{array}$ & $\begin{array}{c}28 / 79(35 \%) \\
P=0.0027\end{array}$ \\
\hline LG & $1 / 51(2 \%)$ & $6 / 51(12 \%)$ & $2 / 51(4 \%)$ & $2 / 51(4 \%)$ & $1 / 51(2 \%)$ & $6 / 51(12 \%)$ \\
\hline
\end{tabular}

HG, high grade; LG, low grade. 

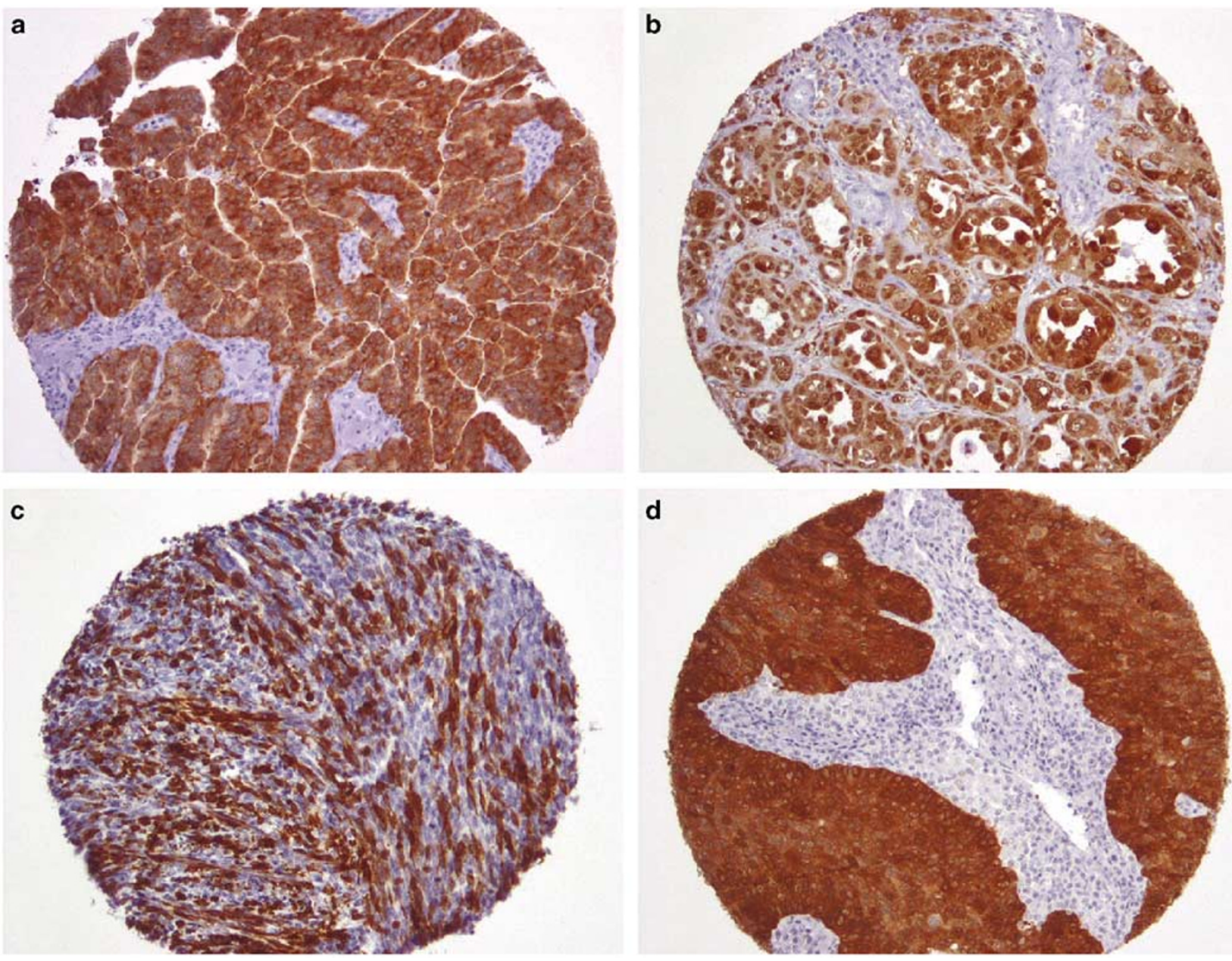

Figure 2 CT antigen expression in high-grade endometrial tumors: M3H67 in serous carcinoma (a); 57B in clear cell carcinoma (b); CT733 in the mesenchymal component of a malignant mixed mullerian tumor (c); ES121 (NY-ESO-1) in a FIGO grade 3 endometrioid adenocarcinoma (d).

\section{WT1 and p53 Expression}

High-grade tumors expressed WT1 significantly more frequently than low-grade tumors $(79 \%$ of all positive cases were high-grade; $P=0.0027)$. Within the high-grade tumors, malignant mixed mullerian tumor expressed WT1 significantly more frequently than all other histologic subtypes $(54 \%$ of all positive cases were malignant mixed mullerian tumors; $P=0.0353)$. Overall expression of WT1 was $26 \%$ (Table 2 ).

High-grade tumors tended to express p53, while low-grade tumors were generally negative $(96 \%$ of all positive cases were high grade; $P<0.001$ ). Malignant mixed mullerian tumors expressed p53 to a greater degree than all other histologic subtypes combined $(62 \%$ of all positive cases were malignant mixed mullerian tumors; $P=0.0004)$. Overall expression was seen in $21 \%$ cases (Table 2).

p53-positive tumors were most likely to coexpress MAGE-A4 $(P=0.0015)$, but an association between WT1 and particular CT antigens was not found.

\section{Discussion}

CT antigens are attractive targets for immunotherapy because of their highly restricted expression in normal tissue and broad expression in a wide range of different tumor types. The present study employs mAbs, which were previously generated by our group and others such as M3H67 to MAGE-A3 (manuscript in preparation), 57B to MAGE-A4, ${ }^{16}$ CT7-33 to CT7, ${ }^{37}$ and ES121 to NY-ESO- $1 .{ }^{23}$ This CTantigen panel has not been previously analyzed in endometrial cancer. However, a cautionary note regarding the fine specificity of these serological reagents, which is best exemplified by $\mathrm{mAb} 57 \mathrm{~B}$, should be made. Although $57 \mathrm{~B}$ was generated as an anti-MAGE-A3 $\mathrm{mAb},{ }^{16,19}$ it was later regarded as a poly-MAGE reagent ${ }^{15,38}$ and is now considered reactive with MAGE-A4. ${ }^{39}$ The problems in defining the specificity of these mAbs may be most likely due to the high degree of homology between several of the CT antigens. However, no immunoreactivity outside the CT antigen spectrum has been reported for the mAbs employed in the current study. 
Nevertheless, their specificity should be regarded with some reservation.

In the present study of endometrial carcinomas, the most prevalent immunoreactivity was found with mAbs M3H67, 57B, and CT7-33, reflecting the expression of MAGE-A3, and MAGE-A4 and CT7, respectively, while NY-ESO-1 expression, as indicated by mAb ES121, was rather low. This expression pattern (ie, common expression of antigens in the MAGE family and low expression of NY-ESO-1) has been found in metastatic melanomas and in carcinomas of the head and neck, breast, and lung in previous studies at the protein level. ${ }^{15,23,37,42,43}$

There is debate regarding the association of CT antigen expression on a protein level and tumor grade. A relationship between MAGE expression and tumor grade has been shown in transitional cell carcinomas of the urinary bladder ${ }^{44}$ and in certain types of brain tumors. ${ }^{45}$ In our analysis, mAb 57B and CT7-33 were statistically significantly more often immunoreactive in high-grade tumors $(95 \%$ of all positive cases $(P=0.001)$ and $89 \%$ of all positive cases $(P=0.0129)$, respectively). $\mathrm{mAb}$ M3H67 was also found to be more frequently expressed in high-grade tumors $(68 \%$ of positive cases) compared with low-grade endometrial carcinomas, although this was not significant (trend, $P=0.0593)$.

With regard to histological subtypes, our analysis showed that 57B immunoreactivity was more often present in papillary serous carcinomas than in other histological subtypes. Also, we did not find any correlation between expression of individual CT antigens. A positive correlation was also found for $p 53$ and 57B immunoreactivity $(P=0.0015)$. No correlation was found between other CT antigens and p53 or between any CT antigen and WT1.

Interestingly, our data contrast partly with those of Resnick et $a l,{ }^{31}$ who analyzed $\mathrm{mAb} 57 \mathrm{~B}$ and $\mathrm{mAb}$ D8.38 (to NY-ESO-1) in endometrial carcinomas. While they found 57B immunoreactivity in $12 \%$ of endometrioid adenocarcinomas, $63 \%$ of papillary serous carcinomas, and $91 \%$ of malignant mixed mullerian tumors, our analysis revealed 57B staining in seven $(8 \%)$ of 85 endometrioid adenocarcinomas, $50 \%$ of papillary serous carcinomas, and $15 \%$ of malignant mixed mullerian tumors. Similar discrepancies were found for NY-ESO-1 expression, which was present in $19 \%$ of endometrioid adenocarcinomas, $32 \%$ of papillary serous carcinomas, and $49 \%$ of malignant mixed mullerian tumors in Resnick's analysis, while our study revealed 6, 6, and $8 \%$ in the respective tumors. However, both studies are similar as to the heterogeneous expression pattern for both antigens. While the discrepancy between Resnick's analysis for mAb 57B in endometrioid adenocarcinomas and ours appears minor, no explanation can be given for the larger discrepancies between both studies in papillary serous carcinomas and malignant mixed mullerian tumors. Previous studies ${ }^{15,23}$ using mAb57B and
anti-NY-ESO-1 mAbs suggest that our findings in endometrial carcinomas are in line with the incidence of expression in other tumors, such as carcinomas of the lung, head and neck, and breast, and certain sarcomas. The expression in endometrial carcinomas appears to be in the mid-range for $\mathrm{mAb} 57 \mathrm{~B}$ and on the lower end for anti-NY-ESO-1 reagents. Immunoreactivity of $91 \%$, as observed by Resnick for mAb 57B in malignant mixed mullerian tumors, appears to be very high. Similar levels of expression have been previously found solely in synovial sarcomas for $\mathrm{mAb} 57 \mathrm{~B}$ and ES121. ${ }^{14,46}$ Further analyses are necessary to resolve the discrepancies observed for immunoreactivity patterns in these studies.

Interestingly, malignant mixed mullerian tumors showed strong immunoreactivity of 57B, CT7-33, and ES121 in both mesenchymal and epithelial neoplastic components. In the other endometrial carcinomas, immunoreactivity was only observed in the malignant glandular component, while the non-neoplastic stromal component was consistently negative. This staining pattern of CT antigen expression in the malignant mesenchymal component of malignant mixed mullerian tumors may be helpful in distinguishing poorly differentiated endometrioid adenocarcinomas from malignant mixed mullerian tumors when the stromal component is not overtly sarcomatous.

The biological significance of CT antigen expression in tumors is still a matter of debate. In malignant melanoma, MAGE protein expression analyzed immunohistochemically with mAb 57B has been shown to correlate only with lymphocytic infiltrates, while no correlation was found with any other prognostic parameter. ${ }^{47}$ On an mRNA level, correlation between CT antigen expression and tumor stage has been reported in several tumors such as renal cell carcinoma, ${ }^{48}$ bladder $^{49}$ and esophageal carcinoma, ${ }^{50}$ and melanoma. ${ }^{51}$ This association has not been demonstrated in others: head and neck carcinoma, ${ }^{52}$ seminoma ${ }^{18}$ stomach, ${ }^{53}$ or colon. ${ }^{54}$ In our present analysis, we did not find any correlation between tumor stage and CT antigen expression, although we documented a relationship between grade and CT antigen expression, particularly for 57B and CT7-33.

In conclusion, the present study showed a rather restricted and mostly heterogeneous pattern of expression of CT antigens in endometrial carcinomas. The highest expression was found for MAGE$\mathrm{A} 3$ and A4. The rather heterogeneous staining pattern in most tumors suggests that the majority of endometrial carcinomas may not be ideal targets for vaccine-based immunotherapy using the antigens analyzed in our study.

\section{References}

1 Boon T, Old LJ. Cancer tumor antigens. Curr Opin Immunol 1997;9:681-683. 
2 Jager E, Gnjatic S, Nagata Y, et al. Induction of primary NY-ESO-1 immunity: CD8+ T lymphocyte and antibody responses in peptide-vaccinated patients with NY-ESO-1+ cancers. Proc Natl Acad Sci USA 2000;97:12198-12203.

3 Stockert E, Jager E, Chen YT, et al. A survey of the humoral immune response of cancer patients to a panel of human tumor antigens. J Exp Med 1998;187: 1349-1354.

4 Van den Eynde BJ, Boon T. Tumor antigens recognized by T lymphocytes. Int J Clin Lab Res 1997;27:81-86.

5 Scanlan MJ, Gure AO, Jungbluth AA, et al. Cancer/ testis antigens: an expanding family of targets for cancer immunotherapy. Immunol Rev 2002;188:22-32.

6 van der Bruggen $\mathrm{P}$, Traversari $\mathrm{C}$, Chomez $\mathrm{P}$, et al. A gene encoding an antigen recognized by cytolytic $\mathrm{T}$ lymphocytes on a human melanoma. Science 1991;254:1643-1647.

7 Traversari C, van der Bruggen P, Luescher IF, et al. A nonapeptide encoded by human gene MAGE-1 is recognized on HLA-A1 by cytolytic $\mathrm{T}$ lymphocytes directed against tumor antigen MZ2-E. J Exp Med 1992;176:1453-1457.

8 Van den Eynde B, Peeters O, De Backer O, et al. A new family of genes coding for an antigen recognized by autologous cytolytic $\mathrm{T}$ lymphocytes on a human melanoma. J Exp Med 1995;182:689-698.

9 Boel P, Wildmann C, Sensi ML, et al. BAGE: a new gene encoding an antigen recognized on human melanomas by cytolytic $\mathrm{T}$ lymphocytes. Immunity 1995;2:167-175.

10 Sahin U, Tureci O, Schmitt H, et al. Human neoplasms elicit multiple specific immune responses in the autologous host. Proc Natl Acad Sci USA 1995;92: 11810-11813.

11 Old LJ, Chen YT. New paths in human cancer serology. J Exp Med 1998;187:1163-1167.

12 Gure AO, Stockert E, Arden KC, et al. CT10: a new cancer-testis (CT) antigen homologous to CT7 and the MAGE family, identified by representational-difference analysis. Int J Cancer 2000;85:726-732.

13 Lucas S, De Smet C, Arden KC, et al. Identification of a new MAGE gene with tumor-specific expression by representational difference analysis. Cancer Res 1998;58:743-752.

14 Antonescu CR, Busam KJ, Iversen K, et al. MAGE antigen expression in monophasic and biphasic synovial sarcoma. Hum Pathol 2002;33:225-229.

15 Jungbluth AA, Busam KJ, Kolb D, et al. Expression of MAGE-antigens in normal tissues and cancer. Int J Cancer 2000;85:460-465.

16 Kocher T, Schultz-Thater E, Gudat F, et al. Identification and intracellular location of MAGE-3 gene product. Cancer Res 1995;55:2236-2239.

17 Dhodapkar MV, Osman K, Teruya-Feldstein J, et al. Expression of cancer/testis (CT) antigens MAGE-A1, MAGE-A3, MAGE-A4, CT-7, and NY-ESO-1 in malignant gammopathies is heterogeneous and correlates with site, stage and risk status of disease. Cancer Immun 2003;3:9.

18 Cheville JC, Roche PC. MAGE-1 and MAGE-3 tumor rejection antigens in human germ cell tumors. Mod Pathol 1999;12:974-978.

19 Hofbauer GF, Schaefer C, Noppen C, et al. MAGE-3 immunoreactivity in formalin-fixed, paraffinembedded primary and metastatic melanoma: frequency and distribution. Am J Pathol 1997;151:1549-1553.
20 Kariyama K, Higashi T, Kobayashi Y, et al. Expression of MAGE-1 and -3 genes and gene products in human hepatocellular carcinoma. Br J Cancer 1999;81: 1080-1087.

21 Chen YT, Scanlan MJ, Sahin U, et al. A testicular antigen aberrantly expressed in human cancers detected by autologous antibody screening. Proc Natl Acad Sci USA 1997;94:1914-1918.

22 Lethe B, Lucas S, Michaux L, et al. LAGE-1, a new gene with tumor specificity. Int J Cancer 1998;76: 903-908.

23 Jungbluth AA, Chen YT, Stockert E, et al. Immunohistochemical analysis of NY-ESO-1 antigen expression in normal and malignant human tissues. Int J Cancer 2001;92:856-860.

24 Rodolfo M, Luksch R, Stockert E, et al. Antigenspecific immunity in neuroblastoma patients: antibody and T-cell recognition of NY-ESO-1 tumor antigen. Cancer Res 2003;63:6948-6955.

25 Eichmuller S, Usener D, Jochim A, et al. mRNA expression of tumor-associated antigens in melanoma tissues and cell lines. Exp Dermatol 2002;11: 292-301.

26 Eichmuller S, Usener D, Thiel D, et al. Tumor-specific antigens in cutaneous T-cell lymphoma: expression and sero-reactivity. Int J Cancer 2003;104:482-487.

27 Odunsi K, Jungbluth AA, Stockert E, et al. NY-ESO-1 and LAGE-1 cancer-testis antigens are potential targets for immunotherapy in epithelial ovarian cancer. Cancer Res 2003;63:6076-6083.

28 Sarcevic B, Spagnoli GC, Terracciano L, et al. Expression of cancer/testis tumor associated antigens in cervical squamous cell carcinoma. Oncology 2003;64: 443-449.

29 van Baren N, Brasseur F, Godelaine D, et al. Genes encoding tumor-specific antigens are expressed in human myeloma cells. Blood 1999;94:1156-1164.

30 Chen YT, Gure AO, Tsang S, et al. Identification of multiple cancer/testis antigens by allogeneic antibody screening of a melanoma cell line library. Proc Natl Acad Sci USA 1998;95:6919-6923.

31 Resnick MB, Sabo E, Kondratev S, et al. Cancer-testis antigen expression in uterine malignancies with an emphasis on carcinosarcomas and papillary serous carcinomas. Int J Cancer 2002;101:190-195.

32 Ozalp S, Yalcin OT, Mete Tanir $\mathrm{H}$, et al. p53 overexpression as a prognostic indicator in endometrial carcinoma. Eur J Gynaecol Oncol 2003;24:275-278.

33 Sorbe B, Risberg B. Prognostic importance of the nuclear proteins p53 and $\mathrm{Rb}$ in conjunction with DNA, nuclear morphometry and grading in endometrial carcinoma. Int J Gynecol Cancer 1997;7:34-41.

34 Salvesen HB, Akslen LA. Molecular pathogenesis and prognostic factors in endometrial carcinoma. APMIS 2002;110:673-689.

35 Makrigiannakis A, Coukos G, Mantani A, et al. Expression of Wilms' tumor suppressor gene (WT1) in human endometrium: regulation through decidual differentiation. J Clin Endocrinol Metab 2001;86: 5964-5972.

36 Dupont J, Wang X, Marshall DS, et al. Wilms tumor gene and p53 expression in endometrial carcinomas: a study of 130 cases using tissue microarrays. Gynecol Oncol (in press).

37 Jungbluth AA, Chen YT, Busam KJ, et al. CT7 (MAGEC1) antigen expression in normal and neoplastic tissues. Int J Cancer 2002;99:839-845. 
38 Rimoldi D, Salvi S, Schultz-Thater E, et al. AntiMAGE-3 antibody 57B and anti-MAGE-1 antibody 6C1 can be used to study different proteins of the MAGE-A family. Int J Cancer 2000;86:749-751.

39 Landry C, Brasseur F, Spagnoli GC, et al. Monoclonal antibody 57B stains tumor tissues that express gene MAGE-A4. Int J Cancer 2000;86:835-841.

40 Kononen J, Bubendorf L, Kallioniemi A, et al. Tissue microarrays for high-throughput molecular profiling of tumor specimens. Nat Med 1998;4:844-847.

41 Hedvat CV, Hegde A, Chaganti RS, et al. Application of tissue microarray technology to the study of nonHodgkin's and Hodgkin's lymphoma. Hum Pathol 2002;33:968-974.

42 Jungbluth AA, Stockert E, Chen YT, et al. Monoclonal antibody MA454 reveals a heterogeneous expression pattern of MAGE-1 antigen in formalin-fixed paraffin embedded lung tumours. $\mathrm{Br} \mathrm{J}$ Cancer 2000;83: 493-497.

43 Juretic A, Spagnoli GC, Schultz-Thater E, et al. Cancer/ testis tumour-associated antigens: immunohistochemical detection with monoclonal antibodies. Lancet Oncol 2003;4:104-109.

44 Kocher T, Zheng M, Bolli M, et al. Prognostic relevance of MAGE-A4 tumor antigen expression in transitional cell carcinoma of the urinary bladder: a tissue microarray study. Int J Cancer 2002;100: $702-705$.

45 Bodey B, Siegel SE, Kaiser HE. MAGE-1, a cancer/ testis-antigen, expression in childhood astrocytomas as an indicator of tumor progression. In Vivo 2002;16:583-588.
46 Jungbluth AA, Antonescu CR, Busam KJ, et al. Monophasic and biphasic synovial sarcomas abundantly express cancer/testis antigen NY-ESO-1 but not MAGE-A1 or CT7. Int J Cancer 2001;94:252-256.

47 Busam KJ, Iversen K, Berwick M, et al. Immunoreactivity with the anti-MAGE antibody 57B in malignant melanoma: frequency of expression and correlation with prognostic parameters. Mod Pathol 2000;13: 459-465.

48 Yamanaka K, Miyake H, Hara I, et al. Expression of MAGE genes in renal cell carcinoma. Int J Mol Med 1998;2:57-60.

49 Patard JJ, Brasseur F, Gil-Diez S, et al. Expression of MAGE genes in transitional-cell carcinomas of the urinary bladder. Int J Cancer 1995;64:60-64.

50 Toh Y, Yamana H, Shichijo S, et al. Expression of MAGE-1 gene by esophageal carcinomas. Jpn J Cancer Res 1995;86:714-717.

51 Brasseur F, Rimoldi D, Lienard D, et al. Expression of MAGE genes in primary and metastatic cutaneous melanoma. Int J Cancer 1995;63:375-380.

52 Kienstra MA, Neel HB, Strome SE, et al. Identification of NY-ESO-1, MAGE-1, and MAGE-3 in head and neck squamous cell carcinoma. Head Neck 2003;25: 457-463.

53 Li J, Yang Y, Fujie T, et al. Expression of the MAGE gene family in human gastric carcinoma. Anticancer Res 1997;17:3559-3563.

54 Hasegawa H, Mori M, Haraguchi M, et al. Expression spectrum of melanoma antigen-encoding gene family members in colorectal carcinoma. Arch Pathol Lab Med 1998;122:551-554. 